\title{
ESTUDIO SOBRE EL FUTURO DE LA CONSTRUCCION*
}

\author{
(STUDY OF THE BUILDING FUTURE)
}

\author{
J. G. Wissema, J. Benes y W. J. Diepeveen
}

070-14

RESUMEN

¿Se producirá, en la próxima década, la virtual desaparición del amianto, cinc y cobre del ámbito de la construcción de edificios? ¿Será el arquitecto, esencialmente, un proyectista industrial? ¿Llegará a ser el trabajo sobre acabados y disposiciones internas el dominio del clienteiconsumidor y, en gran medida, sobre una base de "hágalo por sí mismo»?

Estas son algunas de las indicaciones en un estudio de investigación de las tendencias probables hasta 1990 patrocinado por Dutch Stitching Bouwresearch (SBR) y que ha sido realizado por un comité de 13 especialistas del que forman parte los autores. Las conclusiones, aunque hacen referencia a los Países Bajos, tienen un campo de aplicación más amplio.

\author{
SUMMARY
}

Will the next decade see the virtual disappearance of asbestos zinc and copper from the building scene? Will the architect become essentially an industrial designer? Will work on finishings and internal layouts become the province of the client/customer, much of it on a do-it-yourself basis?

These are some of the indications in a research study of likely trends up to 1990 commissioned by the Dutch Stichting Bouwresearch $(S B R)$ and carried out by a committee of 13 specialists including the authors. Though specifically related to the Netherlands, the conclusions have a broader relevance.
Este informe se centra sobre los resultados del estudio; el método de investigación ha sido ya publicado (ref. 1). Este estudio, patrocinado por la Fundación para la Investigación de la Construcción (SBR) en Rotterdam, investigó que los desarrollos tecnológicos eran más probables durante el período 1975-1990 en el sector de la construcción en los Países Bajos y en qué medida afectarían a la estructura del sector en función de la magnitud relativa de las empresas y las partes implicadas en el proceso de la construcción.

Habida cuenta de que las tendencias tecnológicas son impulsadas por los desarrollos en el mercado, en la sociedad y en la economía mercantil, no se pueden estudiar de forma aislada. Para permitir una investigación de la interacción se utilizó el método del análisis del impacto cruzado. Los datos para este análisis se obtuvieror. mediante entrevistas de expertos en Europa y en Estados Unidos. Las conclusiones, unas doscientas en total, se resumen más adelante. $\mathrm{El}$ informe completo se ha publicado en holandés (ref. 2) y se facilita, a petición, un resumen de 60 páginas en inglés (ref. 3).

El período de 1960 a 1975, que precede al del informe, se caracterizó por el deseo de poner fin a la escasez cuantitativa de viviendas. El desarrollo tecnológico producido en esos años es un fiel reflejo de esta aspiración. Todas las áreas de

- Batiment International. Building Research \& Practice (C/B) September/October 1982. construcción muestran incrementos de escala, prefabricación, mecanización y productividad, con desarrollos inmediatos en materiales y maquinaria. Se produjo un gran aumento en los requisitos y reglamentaciones, dando lugar a procesos de decisión complicados. Se hizo patente un cierto uso de las técnicas de planificación, pero continuó prevaleciendo la improvisación. El pensar en «lo grande» (mayores compañías, mayores contratos) se hizo axiomático.

Se prestó poca atención al ambiente inmediato de viviendas y de edificios, pero, a mediados de este período, surgieron protestas contra las autopistas, edificios de gran altura, proyectos de obras públicas, etc., en un impulso social sobre la edificación que nunca se había visto hasta entonces. Tampoco hubo preparación alguna para la crisis energética ni para un crecimiento económico reducido. También de gran importancia para el sector de la construcción en este período fue el descenso de las expectativas de crecimiento de la población. Finalmente, debemos citar como una tendencia importante la formación de varias grandes compañías que, con sus volúmenes de negocios del billón de dólares, atienden ahora a casi la mitad del mercado interior de la construcción. Una característica de estas compañías es su carácter internacional y su inclinación hacia la integración.

Contra estos antecedentes, las tendencias tecnológicas para el período hasta 1990 pueden agruparse bajo los epígrafes siguientes: 
1. Infraestructura y obras marítimas y en alta mar

2. Cimentaciones

3. Estructuras

4. Acabados

5. Servicios

6. Proyecto y coordinación.

\section{Infraestructura y}

obras marítimas y en alta mar

La impresión general para el período 1975-1990 es de estabilización, si no se produce una recesión en el mercado interior y una expansión de magnitud considerable en el mercado internacional. El trabajo para el mercado interior está experimentando un desplazamiento hacia las obras a menor escala, con consecuencias para la maquinaria requerida. La mecanización es creciente y se hará más uso de modernos equipos de medida.

Las tendencias típicas son:

- virtual desaparición de grandes obras de movimientos de tierras, salvo para construcción de túneles/desmonte para vías férreas (ferrocarriles metropolitanos), obras en canales y proyectos de zonas recreativas;

- aumento de obras más pequeñas, túneles, viaductos, ensanchamiento de carreteras, refuerzo de diques, conductos de agua de refrigeración para calefacción centralizada de barrios o ciudades;

- incrementos considerables en el transporte y transbordo de carbón;

- sustitución/renovación de muchos sistemas de alcantarillado;

- aumento de la tecnología para obras bajo agua;

- nueva generación de grandes equipos de movimientos de tierras para minería de explotación a cielo abierto;

- mayor empleo del método del "escudo» para alcantarillas principales y túneles de vías férreas;

- sustitución de la arena de ríos y pozos por la arena del mar;

- aparición de contratistas especializados para determinadas técnicas de construcción de carreteras;

- el cemento amiantado desaparecerá y no volverá a emplearse;

- el azufre se usará cada vez más como ligante en material de revestimiento.

\section{Cimentaciones}

Los desarrollos habidos pueden devidirse en dos: investigación del suelo y cimentaciones. Se espera que la primera dará lugar a un procedimiento normalizado, mientras que la técnica mejorará

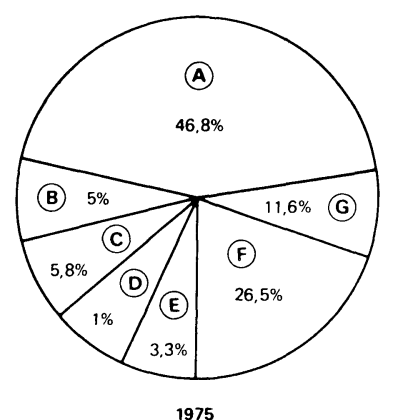

1975

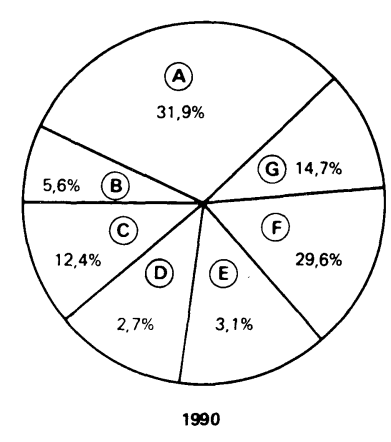

1990
A.-Contratistas. B.-Diseño. C.-Circuito no oficial. D.-Bricolaje E.-Distribución. F.-Prefabricación. G.-Subcontratación.

Desglose porcentual del valor añadido del sector de la construcción en los Paises Bajos, comparando 1975 con la situación probable en 1990

todavía más. Puede preverse mayores

conocimientos de mecánica del suelo, así como una mayor aplicación de la investigación sobre la distribución de esfuerzos como parte de la investigación del suelo.

Las cimentaciones con pilotes continuarán aumentando, en parte porque se utilizarán tipos de suelo todavía peores $\mathrm{y}$, en parte, debido al aumento de la duración de los edificios y viviendas y a la limitación de los gastos de mantenimiento.

Finalmente, se espera que firmas especializadas realizarán tareas específicas de cimentación, tales como renovación o sustitución de cimentaciones en centros urbanos, cimentaciones de caminos, etc. Seguirán los desarrollos en pilotes de tornillo y colados; los de madera dejarán paso a los de hormigón (esbeltos).

Mucho se espera del uso de la microelectrónica, tal como la automatización del pilotaje, medida de la capacidad de carga durante el mismo y mejor disposición del lugar de hinca de los pilotes.

\section{Estructuras}

La perspectiva general es que el hormigón sigue siendo, hasta ahora, el material más importante, pero con un aumento del empleo de las estructuras de acero, de madera y de bloques de carga de mortero de cal, que dan estructuras con un carácter menos uniforme. Los áridos del hormigón habrán de importarse en mayor cantidad, con la consiguiente elevación de los precios.

El hormigón colado tiene posibilidades, pero siempre en combinación con elementos prefabricados, que continuarán siendo predominantes. 
La construcción de edificios será más precisa y el diseño modular se hará dominante en la sustitución de zonas antiguas de las ciudades en formas socialmente aceptables y en nuevos proyectos de edificación, en donde se requiera una gran densidad. La coordinación y normalización modulares, los sistemas abiertos, las técnicas de cubicación, son todas las condiciones necesarias para el logro de estas concepciones. De hecho, se espera que se consiga un gran avance en estas áreas durante el período correspondiente.

Esta forma de trabajar hará posible una separación más estricta entre estructura y los acabados. Esta especialización constituirá una importante ventaja para el cliente y el consumidor, sobre todo considerando el gran incremento del trabajo hecho por el propio usuario. Se prevé un aumento del número de pequeños negocios especializados y más operaciones de subcontratas por el contratista principal. En varios casos pueden presentarse dos clases de contratista: el contratista de estructuras, que subcontrata los trabajos de cimentación y el de trabajos de acabados. Ello es aplicable a la edificación general y a la construcción de viviendas.

\section{Otras tendencias características son:}

- la arena, la grava y la arcilla siguen siendo relativamente baratas y suficientemente disponibles en el ámbito europeo;

- aumento del empleo de tipos modernos de hormigón (nuevos materiales de refuerzo, nuevos ligantes, aditivos, rellenos);

- la cal y los productos cálcicos se mantendrán en su nivel de uso sin incrementos significativos;

- la edificación con sistemas abiertos, empleando elementos normalizados, sustituirá a los sistemas cerrados;

- la construcción con colada in situ y la edificación por elementos se aplicarán en combinación;

- debido a los incrementos en las viviendas de vano único, se aumentará la zona de muros sin soporte de carga;

- incremento de los huecos de tabiquería a $8 \mathrm{~cm}$ para permitir un mayor aislamiento;

- ninguna escasez de madera (aunque posiblemente pueda producirse con carácter temporal en determinadas clases); aumento selectivo en las aplicaciones de la madera;

- el alquiler de maquinaria se hará más frecuente;

- no habrá incrementos adicionales de rendimientos en grúas y dragalinas;

- creciente exactitud y uso de equipos de medida.

\section{Acabados}

La línea principal en estas tendencias es el desarrollo de tecnología que simplifica y hace más baratos los métodos existentes. Tanto es así que cada vez se solaparán más los mercados de profesionales y de aficionados a la construcción. Se prevé que se doble este último mercado.

Los productos especializados se suministrarán directamente por los productores o darán lugar a nuevas actividades de funciones múltiples tales como las de montador/conductor, especialista en revestimientos; jefe de compras/materiales, etc.

Todo ello exige una mayor normalización y un desarrollo adicional de las técnicas de medida. El usuario adquirirá, por sí mismo, los tabiques en un

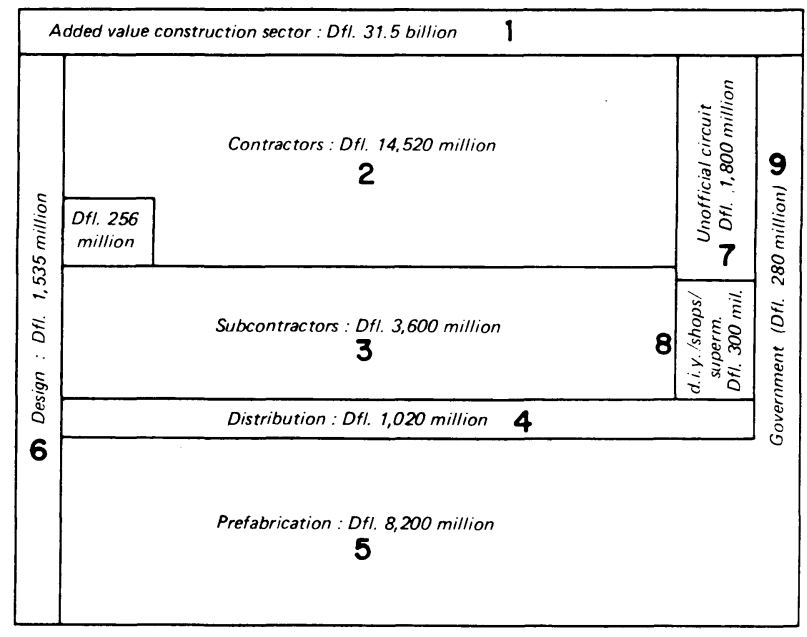

1.- Valor añadido del sector de la construcción. 2.-Contratistas. 3.Subcontratistas. 4.-Distribución. 5.-Prefabricación. 6.-Diseño. 7.Circuito no oficial. 8. - Establecimientos de bricolaje/tiendas/supermercados. 9.-Organismos oficiales.

Valor añadido del sector de la construcción en Holanda en 1975 por las diversas ramas de la industria. Dfl $=$ florines holandeses $(2,6$ florines $=1,0$ dólar aproximadamente)

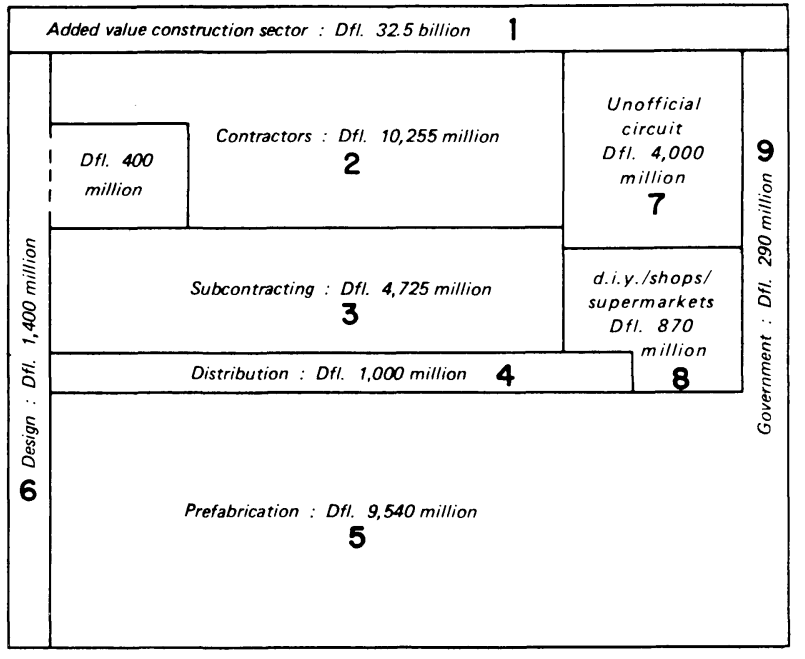

1.- Valor añadido del sector de la construcción. 2. - Contratistas. 3.Subcontratistas. 4.-Distribución. 5.-Prefabricación. 6.-Diseño. 7.Circuito no oficial. 8.-Establecimientos de bricolaje/tiendas/supermercados. 9.-Organismos oficiales.

Forma de distribución probable del valor añadido por diferentes ramas de la industria holandesa de la construcción en 1990: valores a precios constantes de 1975 
almacén o establecimiento especializado en elementos de construcción; la fijación se incluirá en el precio. Lo mismo es aplicable a las puertas de interiores. De hecho, el consumidor individual será capaz de decidir sobre la disposición general de la vivienda, que puede cambiarse, si así lo requiere un nuevo arrendatario. La normalización evitará problemas con las obras de fontanería y acometidas.

Estas tendencias tendrán consecuencias radicales para la estructura comercial de la industria. Por ejemplo, los acabados dejarán el área de la construcción para desplazarse hacia las fornituras.

El trabajo denominado de «a la luz de la luna» (de dedicación parcial) cumple con una importante función, sobre todo en el sector de los acabados. Dicha actividad probablemente llegue a suponer un volumen de negocio de 1-2 billones de dólares. Puede esperarse que los organismos oficiales y la propia industria tratarán de sacar estas actividades del ámbito de la esfera ilegal estimulando a estos trabajadores «piratas» a establecer sus propias empresas.

Se prevé que muchos ejecutivos de empresas de la construcción que hayan estado trabajando en proyectos en países en desarrollo y en el Oriente Medio encontrarán, a su repatriación, problemas de empleo. Es razonable pensar que muchos de ellos establecerán pequeños negocios en la esfera de los acabados.

Veamos un breve resumen de las tendencias en los acabados:

- escasa combinación de funciones con elementos, esto es, sin gran uso de cuartos de baño completos, etc. y sí elementos modulares de construcción;

- gran utilización de revestimientos interiores de hormigón con posibilidades de clavado y aserrado y materiales tipo sandwich:

- mayor racionalización y mejores líneas de las herramientas manuales y motorizadas;

- mayor fusión de los mercados de profesionales y de aficionados a la construcción, en lo que concierne a las herramientas;

- empleo de paredes interiores desmontables y económicas con buenas propiedades acústicas;

- uso más sofisticado de plásticos y de resinas;

- las unidades de neopreno/vidrio se harán más frecuentes;

- el empleo de aluminio se limitará a marcos y cableado;

- los suelos de madera tienden a desaparecer;
- creciente uso de técnicas y materiales de aislamiento.

\section{Servicios}

Este sector ya está muy racionalizado con una proporción relativamente alta de materiales fácilmente aplicables, conexiones más simples y un carácter prefabricado considerable. Muchos de los cambios previstos están ligados con los más altos costes de la energía y, sobre todo, con la desaparición del gas natural como combustible barato. La energía solar, las bombas de calor y la calefacción centralizada de barrios y ciudades harán su aparición generalizada, de modo que el agua rara vez se calentará por encima de una temperatura de $60{ }^{\circ} \mathrm{C}$. Esta temperatura y los desarrollos en la calidad de la vivienda y en el aislamiento de los edificios darán lugar a sistemas de calefacción completamente distintos.

El mayor aislamiento de las viviendas y de otros edificios acelerará el desarrollo de sistemas de ventilación (automatizados). No se prevé la continuidad del aire acondicionado.

El trabjo por cuenta del propio usuario se hará frecuente en el sector de los servicios, al posibilitarse por conexiones y conductos flexibles y las abrazaderas, deslizaderas y juntas encoladas. No se espera la aparición de sistemas revolucionarios de eliminación de residuos. Los sistemas antirrobos y contraincendios se harán comunes; está prevista una normalización. El cobre y el plomo desaparecerán de la construcción de edificios.

\section{Proyecto y coordinación}

Parece existir una gran variedad de funciones a cumplir por el arquitecto. En la realización de proyectos, está previsto que el trabajo del arquitecto estará mucho más influido por la edificación en sistemas abiertos y por la disponibilidad de elementos normalizados de una edificación. No se prevé que el arquitecto llegue a hacerse una especie de director de construcciones; por el contrario, se espera un papel más limitado como supervisor a pie de obra. Ello significa que el arquitecto se hará más bien un proyectista industrial.

Además de lo anterior, se ve al arquitecto como la guía principal en el ámbito de leyes y reglamentaciones. Muchos arquitectos encontrarán su función en el proyecto de la «estructura de fábrica» o medio ambiente de la edificación; quienes estén al servicio de contratistas o proveedores de construcciones se concentrarán en el proyecto de elementos de edificios normalizados, con el empleo de muchos materiales nuevos que irán apareciendo. También otros se especializarán 
como diseñadores de elementos de acabado, posiblemente junto con la función de coordinador de los mismos. Pocos arquitectos, de los que ahora están realizando sus estudios, llegarán a desempeñar la profesión en la forma clásica.

\section{Interés por la calidad}

Sí puede decirse que el período de la postguerra, sobre todo 1960-1975, se caracterizó por intentar resolver el problema de la escasez cuantitativa de viviendas; en el período de 1975 a 1990 se vaticina que el énfasis estará en la solución de una escasez cualitativa de viviendas, mientras que, en el período anterior, el control de costes hubo de incidir en incrementos en escala de producción, tanto en el sentido técnico como en el organizativo; en el período venidero, el control de costes se basará cada vez más en la normalización y en la prefabricación asociada, precisión en la construcción, disminución de la mano de obra in situ y también más precisión en la planificación y coordinación. Esta tendencia conduce a un incremento de escala para la industria proveedora y a la especialización y descentralización en la construcción, con más subcontratas. Una excepción es el lado internacional del sector de los movimientos de tierras y obras en alta mar, en donde los desarrollos parecen dirigirse hacia una continuidad de la integración.

El empleo de materias primas en la construcción de edificios experimentará muy pocos cambios. Arena, grava, cemento y arcilla seguirán siendo las materias primas básicas $\mathrm{y}$, en la posición relativa del hormigón, se espera poco desplazamiento de ladrillos y bloques de cal y arena. La expectativa de que las estructuras metálicas de acero y que en menor medida, los armazones de madera encontrarán más aplicación en algunos sectores del mercado, no se contradice con lo anteriormente expuesto. Nuevos áridos y ligantes se utilizarán también en el hormigón.

La madera se aplicará más en el ámbito decorativo con hasta $3 \mathrm{~m}^{3}$ por vivienda y tendrá más uso en los edificios de oficinas y de fábricas que en la actualidad. Diferentes calidades de madera se emplearán más selectivamente que en el momento presente. Desaparecerán algunos materiales en los edificios tales como amianto, plomo, cinc y cobre. La aplicación de los plásticos se hará con más inteligencia; es decir, se aprovecharán mejor las características específicas de los plásticos. Entre otras acciones, se les mezclará con los materiales tradicionales.

Un material relativamente nuevo que continuará su avance en combinación con otros (hormigón, plásticos), así como por sí mismo, es la lana de vidrio. No se trata de que se desplace a los materiales tradicionales por el plástico; las «casas del futuro» no se construirán sobre una base a gran escala y no merecen tal calificativo. El desarrollo en la aplicación de los plásticos presenta un cierto paralelismo con el de la industria del automóvil, en donde no se ha llegado a fabricar un coche de plástico.

En la técnica de la edificación, el mayor cambio se espera ciertamente en los acabados estructurales como especialización en la construcción. Habrá un efecto de gran influencia en el sector de los acabados. Los servicios cambiarán radicalmente debido a la aparición de nuevas técnicas de calefacción y aislamiento y a la mayor simplificación de técnicas existentes de nuevas conexiones, con la más amplia aplicación de conexiones flexibles. El sector de la cimentación cambiará radicalmente debido a la mayor aplicación de la mecánica del suelo, de pilotaje de mayor precisión, de muchas más técnicas de cimentación y de más supervisión de la propia cimentación. En el sector de las infraestructuras y de los movimientos de tierras no se esperan muchos cambios, aparte de las obras en alta mar y con la excepción de la creciente aplicación de sencillos equipos auxiliares de precisión, tales como aparatos laséricos.

Respecto a la maquinaria, la tendencia es hacia equipos móviles y más pequeños, con funciones múltiples y con unas prestaciones no muy diferentes a las actuales, pero con una mayor capacidad de desplazamiento. Con un aumento en el alquiler de maquinaria se conseguirá más flexibilidad de uso de la misma. Solamente en la minería de explotación a cielo abierto y en alta mar puede esperarse incrementos de gran magnitud en la maquinaria.

\section{Influencias del mercado y sociales}

La influencia del usuario y del cliente de construcciones continuará aumentando, incluso en el caso de servicios, porque hay más opción de sistema de calefacción, aislamiento, suministro de agua caliente y canalizaciones. Por otra parte, se espera que las exigencias oficiales seguirán aumentando. La creciente influencia del control oficial y del comprador llevará necesariamente al desarrollo de componentes modulares y especificaciones de productos funcionales (termorresistencia, resistencia acústica, etc.).

Disminuirá la influencia del arquitecto diseñador de edificios sobre la especificación del producto, porque cada vez dependerá más de lo que ofrezca la industria proveedora en la forma de módulos normalizados (sistemas abiertos). Ello puede evocar imágenes de ciencia ficción, pero no hemos de olvidar que actualmente hay una gran variedad de tejidos, muebles, vehículos y otros productos, todos ellos de producción a gran escala, que anteriormente tenían un objetivo muy distinto.

$\mathrm{Si}$, actualmente, las especificaciones del producto inciden, sobre todo, en el ámbito de la estética, en el futuro también habrá criterios «rigurosos» para 
las características físicas, flexibilidad, durabilidad y fiabilidad, así como para los costes, que se harán de carácter crucial. Con el empleo de programación de ordenador, el proyectista será capaz de calcular, para el futuro usuario, la diferencia en el coste para diversos cambios en el diseño. Los programas, mediante los cuales el usuario consigue una idea global del interior del edificio en la pantalla, implicará más al usuario en el proceso de diseño.

\section{El problema de innovación}

La creciente variedad, cambio e interacciones de todos los participantes del proceso de construcción exigen innovaciones de gran magnitud en los procesos de toma de decisiones y en la organización y cooperación dentro del sector. No obstante, la innovación, que es la aplicación satisfactoria de algo nuevo, parece más remota que nunca. La tecnología está allí y la necesidad está allí. ¿En qué radica, entonces, las dificultades? ¿Cuáles son los factores que fomentan o impiden la innovación?

- Los sistemas cerrados impiden la innovación porque las series son demasiado pequeñas para ser capaces de soportar el riesgo de innovación.

- La venta de un producto, en lugar de una función, obstaculiza la innovación y viceversa. $\mathrm{Si}$ se vende capacidad de soporte de carga en lugar de pilotes de forma habitual, entonces, la innovación se estimulará en el sector de la cimentación.

- En términos más generales, la separación de quienes soportan los costes y riesgos de aquellos que se benefician de las ventajas de la innovación resulta desastrosa para el progreso de la innovación. $\mathrm{Si}$, por ejemplo, un proveedor inventa un nuevo elemento de tejado termoaislante que comienza a alabearse transcurridos unos pocos años, entonces, el contratista que ha de «pagar el pato» no encontrará facilidades para ser compensado, a su vez, por el proveedor.

- La innovación a pequeña escala se probará que es más importante que la innovación a gran escala, en el sector de la construcción. La falta de consideración de esto último resulta costosa por lo que el riesgo es grande e incalculable (compárese con los aditivos de acortamiento del tiempo de fraguado en el hormigón que se encontraron al abordar el refuerzo de armadura). El riesgo implicado en una nueva clase de revestimiento exterior es considerablemente menor mientras que sigue siendo más limitado el de un nuevo tipo de puerta de interior.

- Los riesgos en la maquinaria pueden hacerse menores alquilando en lugar de comprar. El alquiler en vez de adquirir fomenta, pues, la innovación.

- La construcción orientada hacia el mercado (construcción según las necesidades) fomenta la innovación; el sistema de construcción de protección oficial la retarda. La implicación de los usuarios estimula la innovación porque muchos de ellos desean lo más reciente en confort, modo, precio o a causa de la novedad en sí misma. El consumidor es, a largo plazo, la fuente de toda innovación.

- La construcción en serie, que es la forma más primitiva de ampliación de escala, tiene, de hecho, como consecuencia una onda de innovación. La normalización a más pequeña escala (coordinación modular) llevará también a una onda de innovación.

- El control/inspección de calidad por un organismo neutral mejora la innovación.

- Un dispositivo no caro de control de calidad, que puede utilizarse por personas no especializadas, ayudará a la innovación.

\section{Tendencias en la estructura de la industria}

Después del resumen de las tendencias tecnológicas, las conclusiones principales acerca de los futuros desarrollos estructurales del sector de la construcción son:

- Un aumento de la especialización de las empresas, sobre todo en las operaciones de acabado.

- Descentralización en las empresas contratistas.

- Más subcontratación, que da lugar a reducciones en las plantillas de mano de obra de contratistas importantes. Durante el período de 1975 a 1990, el valor añadido de contratistas disminuirá del $47 \%$ al $32 \%$ del valor añadido total del sector; el valor añadido de los subcontratistas se espera que se eleve del $12 \%$ al $15 \%$.

- Aumento de las actividades de «hágalo por sí mismo», aparición de un trabajo por aficionados de segunda generación, esto es, trabajos más complicados se ponen al alcance de las capacidades de los profanos, tanto hombres como mujeres. El valor añadido aumentará del $1 \%$ al $3 \%$ en el período de 1975 a 1990.

- Además de los canales comerciales tradicionales, surgirán establecimientos de bricolaje y canales comerciales directos de proveedor a contratista. 
El valor añadido del sector de distribución mostrará un decrecimiento porcentual.

- Las oficinas técnicas de proyectos mantendrán su posición con un desplazamiento de sus actividades hacia las partes implicadas en la prefabricación y las fases de construcción. Los arquitectos tenderán a hacerse proyectistas industriales y especialistas en reglamentaciones y planificación. El sector de proyectos experimentará un pequeño incremento en el valor añadido porcentual.

- El trabajo se desplazará desde el emplazamiento de la construcción a la fábrica. La prefabricación totalizará un $30 \%$ del valor añadido del sector en 1990, en comparación con el $26 \%$ en 1975 .
- El circuito no oficial (mercado negro, «trabajos de pluriempleados») puede doblarse del $6 \%$ al $12 \%$ del valor añadido del sector, a no ser que se tomen medidas drásticas.

\section{Referencias/Bibliografía}

1. WISEMA, J.E. y BENES, J., «A cross impact case study -the Dutch construction sector» (Un estudio de caso de impacto cruzado- el sector de la construcción en Holanda), Futures, octubre 1980, p. 394-404.

2. La Foundation for Construction Research (SBR), un centro de investigación independiente que trabaja, en gran medida, sobre una base contractual.

3. De Stitching Bouwresearch, PO Box 20740, 3001 JA Rotterdam, Países Bajos.

publicaciones del i.e.t.c.c.

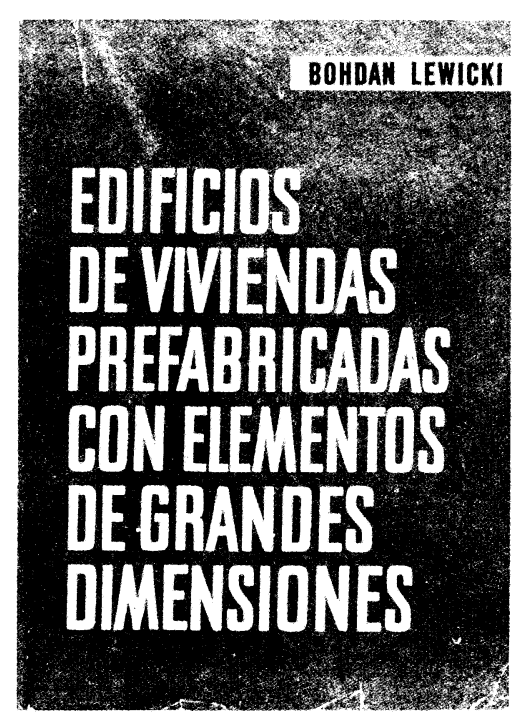

Bohdan Lewicki

Este libro trata de los problemas relativos a la construcción de los edificios de viviendas o publicos realizados con elementos prefabricados de grandes dimensiones. Se han estudiado los problemas de arriostramiento así como los que plantea la resistencia de los elementos y de la estructura; se han examinado las cuestiones de orden higrotérexaminado las cuestiones de orden higr mico, acústico y de resistencia al fuego también se ha profundizado en el estudio de la estanquidad de los muros exteriores y de las juntas.

La obra incluye numerosas ilustraciones que dan detalles de diversas soluciones, asi como ejemplos de cálculo, tablas de valores numéricos, diagramas y ábacos.

Un volumen encuadernado en tela, de $24 \times 17 \mathrm{~cm}$, compuesto de 616 págs.

Precios: 2500 ptas : SUSA 36.00
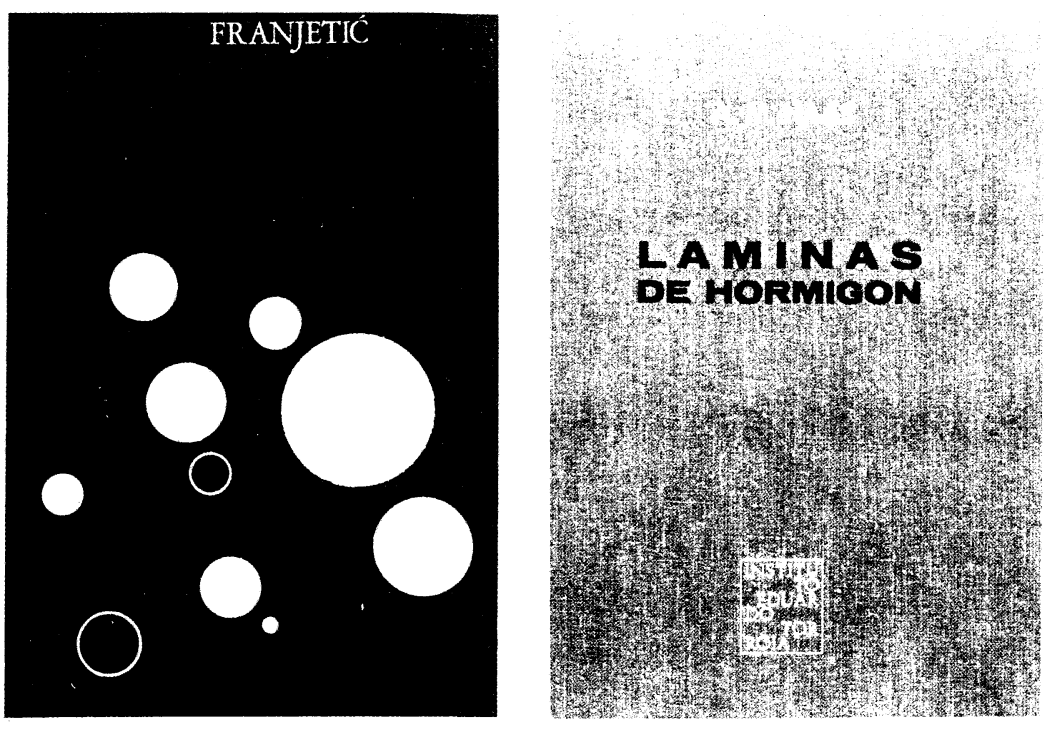

Zorislav Franjetić

En la obra de Franjetić se expone de una forma minuciosa, ordenada y sistemática, forma minuciosa, ordenada y sistemática, nocimiento actual sobre el endurecimiento rápido del hormigón. Parte el autor de los principios básicos y llega a las últimas consecuencias y realidades técnicas y economicas.

Es una obra de consulta, tanto para el investigador sobre la materia, como para el proyectista y el realizador y montador de plantas e instalaciones y equipos de curado y endurecimiento rápido.

Un volumen encuadernado en cartóné, de $17 \times 24,5 \mathrm{~cm}$, compuesto de 385 págs. 110 figuras y 10 tablas.

Precios: 2.500 ptas.; \$USA 36.00

\section{A. M. Haas}

Al escribir este libro el autor intentó poner a disposición de los estudiantes y de los ingenieros unos conocimientos prácticos, adecuados para servir de guia en el diseño y construcción de láminas delgadas de hormigón.

El autor está convencido de que el éxito en el diseño de una lámina exige, por parte del proyectista, un examen de las tres fases por las que pasa la materialización de la lámina: el diseño, el análisis estructural y la construcción de la estructura.

Un volumen encuadernado en tela, de $17 \times 24,5 \mathrm{~cm}$, compuesto de 420 págs., 141 figuras, 22 fotografias y 6 tablas.

Precios: 2.500 ptas.; \$USA 36.00 